\title{
Follow-up, surgery and proton beam therapy \\ for a pituitary sella chondrosarcoma
}

Yasir Mohamed Elhassan ${ }^{1}$, Bernie Foran², Tim Hodgson ${ }^{3}$,

Paul Ince ${ }^{4,6}$, Saurabh Sinha ${ }^{5}$ and John Newell-Price ${ }^{1,7}$

Depts of ${ }^{1}$ Endocrinology, ${ }^{2}$ Oncology, ${ }^{3}$ Neuroradiology, ${ }^{4}$ Neuropathology and ${ }^{5}$ Neurosurgery, Pituitary MDT, Royal Hallamshire Hospital, Sheffield Teaching Hospitals NHS Foundation Trust, Sheffield, UK; ${ }^{6}$ Sheffield Institute for Translational Neurology Research (SITraN) and Dept of ${ }^{7}$ Human Metabolism University of Sheffield, UK

\section{Introduction}

We report a case of sellar chondrosarcoma presenting as a non-functioning pituitary tumour

\section{Case History}

- 40 year old female referred with nine months history of secondary amenorrhoea and galactorrhoea

- She was otherwise well with no other medical history

- Not on regular medications

- At presentation, examination was normal

- Prolactin 2000mU/L (NR 86-324)

- Normal FBC, U\&E, LH, FSH, TSH, FT4, IGF-1 and short Synacthen test

\section{Imaging}

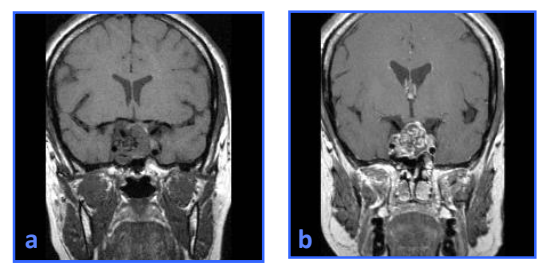

Pituitary MRI showing a 30mm sellar mass (a) Pre-Gadolinium (b) Post-Gadolinium

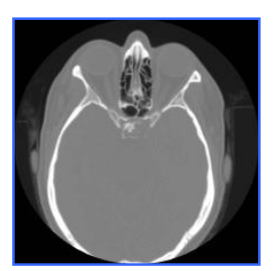

CT scan showing calcification in the sella

\section{Case Progress}

- Presumed Craniopharyngioma in view of calcification

- Surgery was recommended but the patient declined

- Opted for a period of radiological surevillance

- Commenced on Cabergoline; achieved normal prolactin and regular periods and galactorrhoea resolved

\section{Follow-up Imaging}

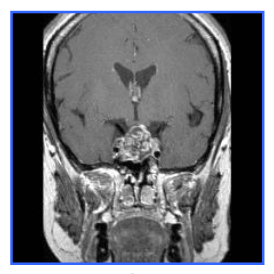

Initial MRI

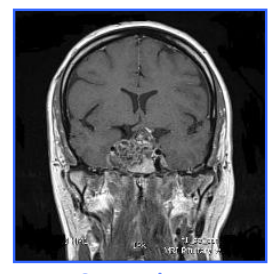

3 years later

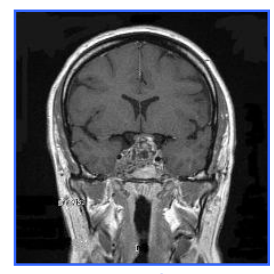

A year later

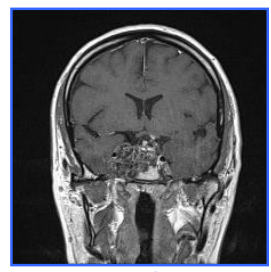

4 years later

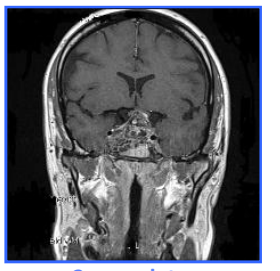

2 years later

Serial MRI scanning showing slow increase in the size of the sellar mass whilst she remained reluctant to have surgery. The patient gradually developed right $6^{\text {th }}$ and left $4^{\text {th }}$ cranial nerve palsies. 4 years later she agreed to undergo surgery

\section{Further Management}
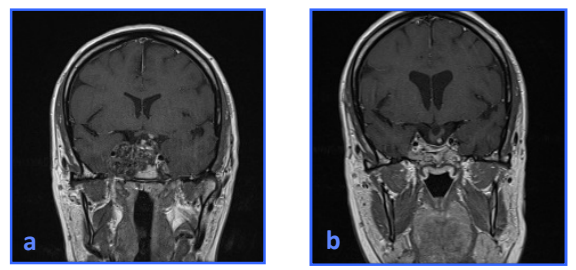

Pituitary MRI (a) Pre- and (b) postendoscopic transsphenoidal debulking with complete resection of the suprasellar component. There is residual tissue in the right cavernous sinus. Pituitary function remains intact

\section{Histopathology}

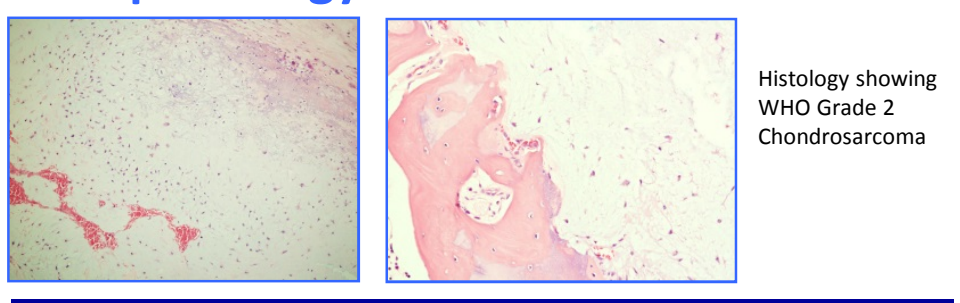

\section{Proton Beam Therapy}

- Referred for Proton Beam therapy in Florida, USA, which was well tolerated
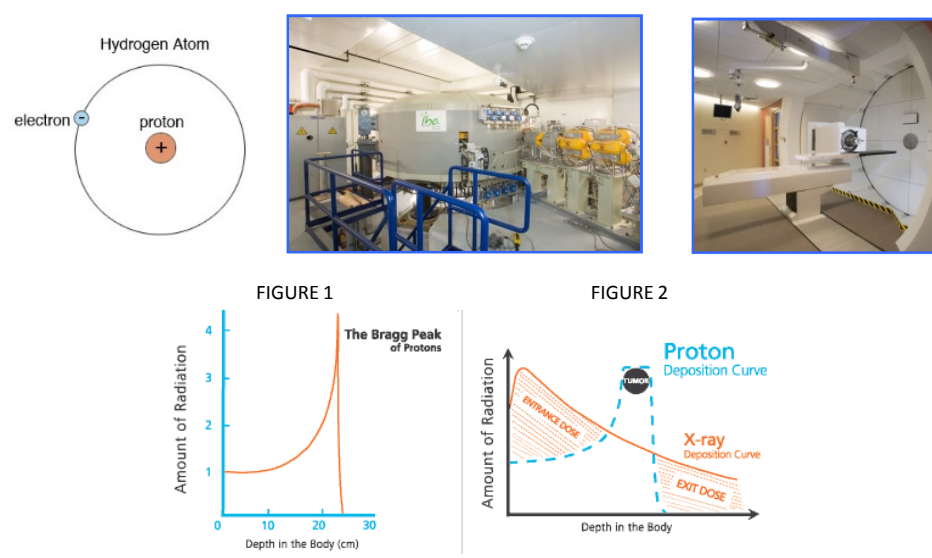

FIGURE 2

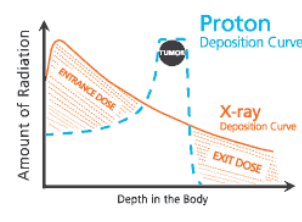

www.floridaproton.org
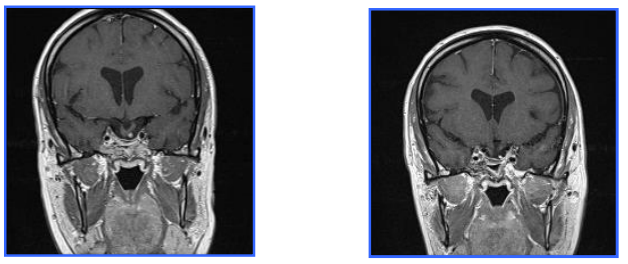

Post-operative MRI

6 months Post-proton therapy

\section{Discussion}

- Pituitary chondrosarcomas are rare tumours of cartilage-forming cells arising from the sella, usually presenting as a non-functioning sellar mass

- The most important predictor of good long-term outcome is the extent of resection of the initial tumour

- However, the anatomical location of these tumours may render complete resection extremely difficult and hazardous to achieve, and proton beam therapy is recommended for any residual

- This case demonstrates that these tumours are slow growing

- The case also illustrates the importance of histological diagnosis for proton therapy to be offered which is recommended for any residual as it allows safer delivery of significantly higher radiation doses 